\title{
Análisis de la Supervivencia de los pacientes con insuficiencia cardiaca en la Sierra Norte de Ecuador entre los años 2015 y 2020
} Survival analysis of patients with heart failure in the Ecuadorian Sierra Norte between 2015 and 2020

Resumen: La insuficiencia cardíaca constituye una de las causas más importantes de morbimortalidad a nivel mundial. En países en vías de desarrollo existe escasa evidencia respecto al pronóstico de esta enfermedad. Por este motivo el presente estudio tiene como objetivo establecer las causas más importantes de insuficiencia cardíaca y determinar la supervivencia en función de su etiología en pacientes diagnosticados con insuficiencia cardíaca en un Hospital General de segundo nivel ecuatoriano. Se efectuó un estudio mono céntrico, retrospectivo, de prevalencia, en 284 pacientes ambulatorios, con diagnóstico de insuficiencia cardíaca, durante los años 2015-2020. Las diferencias entre los subgrupos se analizaron estadísticamente mediante pruebas de ANOVA y se realizó el análisis de supervivencia por el método actuarial y regresión de Cox. La principal causa de insuficiencia cardíaca fue la hipertensiva (55,5\%), seguida de la etiología isquémica (20,8\%). El análisis de supervivencia evidenció que la sobrevida global a 5 años fue del 17\%, con tiempo promedio de vida de 3,46 años. La principal causa de insuficiencia cardíaca entre los pacientes fue la hipertensiva y la supervivencia global a 5 años es inferior a la reportada en otros estudios.

Palabras clave: hipertensión arterial, insuficiencia cardíaca, sobrevida.

Abstract: Heart failure is one of the most important causes of morbidity and mortality worldwide. In developing countries there is little evidence regarding the prognosis of this disease. For this reason, the present study aims to establish the most important causes of heart failure and determine survival based on its etiology in patients diagnosed with heart failure in a second-level Ecuadorian General Hospital. A retrospective, single-center, prevalence study was conducted in 284 outpatients, diagnosed with heart failure, during the years 2015-2020. The differences between the subgroups were statistically analyzed using ANOVA tests. Survival analysis was performed using the actuarial method and Cox regression. The main cause of heart failure was hypertensive (55.5\%), followed by ischemic etiology (20.8\%). Survival analysis showed that overall 5-year survival was $17 \%$, with an average life time of 3.46 years. The main cause of heart failure among patients was hypertensive and the 5-year overall survival is lower than that reported in other studies.

Key words: arterial hypertension, heart failure, survival.

\section{Introducción}

La insuficiencia cardíaca constituye una de las causas más importantes de morbimortalidad a nivel mundial $(1,2)$. Se calcula que esta enfermedad afecta entre el $2 \%$ y el $5 \%$ de la población en general, lo que equivaldría a 37,7 millones de personas ${ }^{1-3}$.

En los Estados Unidos de América una de cada ocho defunciones es atribuidas de manera directa o indirecta al diagnóstico de insuficiencia cardíaca. Mientras que en la Unión Europea se estima que hasta el $40 \%$ de los decesos reportados se deben a esta patología 2,4 .

Actualmente, esta enfermedad es una de las más letales al compararse con algunos de los tipos de cáncer más comunes lo que hace prever que su prevalencia aumente en un $46 \%$ hasta el año 2030 3.4.

En lo que respecta a costos, se calcula que la insuficiencia cardíaca, a nivel mundial, representa un gasto anual de 108 billones de dólares, lo que constituye el $60 \%$ del presupuesto en salud destinado a los cuidados directos o indirectos de los pacientes con esta enfermedad ${ }^{3,5}$.

En Ecuador existe poca evidencia sobre la epidemiología de la insuficiencia cardíaca. De ahí que este estudio pretende determinar: (i) las causas más importantes de insuficiencia cardíaca y (ii) la supervivencia global y en función de la etio- logía de los pacientes en control ambulatorio con diagnóstico previo de esta enfermedad.

\section{Materiales y métodos}

\section{Diseño del estudio}

Se realizó un estudio mono céntrico, transversal, retrospectivo en el Hospital General San Vicente de Paúl de la ciudad de Ibarra, Ecuador. Para esto se analizaron las historias clínicas de los pacientes diagnosticados con insuficiencia cardíaca entre enero de 2015 y enero de 2020.

\section{Población de estudio}

La identificación de los casos se realizó a través de las historias clínicas definidas según la Clasificación Internacional de Enfermedad CIE-10 con los códigos I-50, I-51, I-52, I-23, I-42 que corresponden a: (i) insuficiencia cardíaca, (ii) complicaciones y descripciones mal definidas de enfermedad cardíaca, (iii) otros trastornos cardíacos en enfermedades clasificadas en otra parte, (iv) ciertas complicaciones actuales seguidas de un infarto agudo de miocardio y (v) cardiomiopatía, respecti-

Departamento de Medicina Interna, Hospital San Vicente de Paúl, Ibarra, Ecuador.

${ }^{2}$ Escuela de Ciencias Biológicas e Ingeniería, Hacienda San José s/n, Urcuquí, Ecuador 
vamente.

En total se identificaron 300 historias clínicas de las cuales se incluyeron 284. No formaron parte de este estudio los pacientes: (i) menores de 18 años de edad, (ii) con diagnóstico de insuficiencia cardíaca secundaria a cardiopatía congénita y (iii) con registros de información incompleta.

El estudio cumplió con la aprobación del Departamento de Docencia del Hospital San Vicente de Paúl en concordancia con los requerimientos bioéticos estipulados en la declaración de Helsinki.

\section{Parámetros clínicos, laboratoriales y ecocardiográficos}

A partir de los valores de: (i) presión arterial sistólica, (ii) presión arterial diastólica, (iii) presión arterial media, (iv) presión de pulso, (v) frecuencia cardíaca y de los datos de laboratorio se obtuvo el promedio de cada una de estas mediciones que se determinaron durante los controles ambulatorios.

La clase funcional se la sistematizó de acuerdo a la clasificación de la New York Heart Association (NYHA). El valor del filtrado glomerular se calculó mediante la fórmula propuesta por la Chronic Kidney Disease Epidemiology Collaboration CKD-EPI, y el de la fracción de eyección se determinó a través del método de Simpson permitiendo clasificar los grupos en función de los criterios de la Sociedad Europea de Cardiología.

\section{Análisis estadístico}

El análisis de las variables cuantitativas se realizó mediante medidas de tendencia central y de dispersión. Las variables cualitativas se expresaron como porcentajes. La comparación de los diferentes subgrupos se realizó mediante ANOVA de un factor y el análisis post hoc se efectuó a través las pruebas de Scheffe o de Games-Howell según corresponda.

El análisis de supervivencia se elaboró por el método actuarial seguido de la regresión de Cox. Los valores $p<0,05$ fueron considerados como significativos con intervalos de confianza a un nivel de certeza del 95\%. El procesamiento de los datos se efectuó con el paquete estadístico SPSS v.22 (IBM Corporation)

\section{Resultados}

\section{Características basales de los pacientes}

Se analizaron 284 historias clínicas de pacientes procedentes de consulta externa con diagnóstico de insuficiencia cardíaca. El promedio de edad de los sujetos fue de 75,2+/13 años. Del total de los pacientes, el 49,3\% fueron del sexo femenino (Tabla 1). La autodefinición étnica mayoritaria fue mestiza $(73,9 \%)$ y la mayoría de pacientes indicaron residir en la provincia de Imbabura $(70,4 \%)$ (Tabla 1$)$.

Al momento del diagnóstico, la mayor parte de los sujetos se encontró en las clases funcionales II (36\%) y III (36,7\%) según la clasificación de la NYHA, siendo la etiología más común la hipertensiva (55,5\%). El tratamiento de estos pacientes mayoritariamente fue con base a: (i) los inhibidores de la enzima convertidora de angiotensina / antagonistas del receptor de la angiotensina tipo 2, (ii) diuréticos de asa, (iii) betabloqueantes y (iv) antagonistas de la aldosterona (Tabla 1).

El 49,5\% de los pacientes fue catalogado con insuficiencia cardíaca con fracción de eyección reducida con promedio de filtrado glomerular correspondiente al estadio IIIA de la clasificación Kidney Disease Improving Global Outcomes (KDIGO).

Otros hallazgos de importancia fueron la presencia de hiponatremia leve, valores elevados de péptido cerebral natriu- rético (proBnP) y la presencia de hipocolesterolemia. (Tabla 1)

\section{Relación entre la etiología y las características basales de los pacientes con insuficiencia cardíaca}

El análisis de la etiología de la insuficiencia cardíaca en función de los subgrupos y en relación de las variables obtenidas de las historias clínicas de los pacientes observó que: (i) la edad, (ii) la presión arterial sistólica, (iii) la presión arterial diastólica, (iv) la presión arterial media, (v) la presión de pulso, (vi) los niveles de colesterol (LDL y HDL) y (vii) los triglicéridos mostraron diferencias estadísticamente significativas. (Tabla 2).

El análisis post hoc demostró que la presión arterial sistólica, la presión arterial media y la presión de pulso presentaron diferencia estadísticamente significativa $(p<0,05)$ en función de las etiologías hipertensiva y desconocida de la insuficiencia cardíaca (Tabla S1). Además, los niveles de triglicéridos tuvieron diferencia significativa $(p<0,05)$ entre los subgrupos en función de la causa isquémica respecto a la causa desconocida. Al igual que el colesterol LDL fue significativamente diferente $(p<0,05)$ en relación a la causa isquémica frente a la etiología alcohólica (Tabla S1).

\section{El diagnóstico de insuficiencia cardíaca disminuye la supervivencia de los pacientes}

El análisis de supervivencia global de los pacientes con insuficiencia cardíaca entre los años 2015 y 2020 mediante el método actuarial fue del $17 \%$ a los 5 años a partir del diagnóstico (Figura 1).

Según la etiología de la enfermedad y el tiempo de sobrevida encontramos que hubo diferencias entre ellas. Así, la supervivencia de los pacientes con insuficiencia cardíaca debida a hipertensión arterial fue de 3,49 años (IC95\%; 3,38-4,51) mientras que para la asociada con etiología isquémica fue, de 4,19 años (IC95\%; 2,73-5,64). Los pacientes con insuficiencia cardiaca de etiología valvular tuvieron 4,35 años (IC95\%; 2,696,01 ) de sobrevida y los de origen alcohólico 4,80 años (IC95\%; $3,89-5,81$ ). Los pacientes con insuficiencia cardíaca de causa desconocida y con enfermedad debida a otros motivos sobrevivieron 2,87 años (IC95\%; 2,25-3,49) y 3,42 años (IC95\%; 2,014,83), respectivamente (Figura 2).

El análisis de regresión de Cox evidenció que el menor número de hospitalizaciones (HR: 0,5; IC95\%; 0,45-0,703; $p=$ 0,000) y la etiología hipertensiva (HR: 0,56; IC95\%; 0,34-0,910; $p=0,019)$ tuvieron efecto protector. Por otro lado, el valor del desplazamiento sistólico del plano del anillo tricuspídeo (TAPSE) fue < 16 mm (HR: 1,01; IC95\%; 1,005-1,026; $p=0,004$ ) lo que se asoció con mayor mortalidad. $(X 2=59,2 ; p=0,000)$.

\section{Discusión}

La insuficiencia cardiaca es una dolencia que afecta de manera especial a personas mayores de 65 años. En nuestro estudio la media de edad de los pacientes fue de entre 70 y 80 años. Este resultado es semejante a los publicados en estudios anteriores ${ }^{1,5-7}$. Sin embargo, en cuanto al sexo de los pacientes encontramos un predominio de hombres con insuficiencia cardiaca a diferencia de lo publicado anteriormente ${ }^{1,7,8}$ (Tabla 1).

La etnia es un factor determinante para la insuficiencia cardíaca. Se sabe que existe mayor predisposición y peor pronóstico de vida en personas de etnia negra que en blancos e hispanos ${ }^{3}$. En este estudio, el mayor porcentaje de personas fueron mestizos, seguidos de los indígenas y de los negros (Tabla 1 ).

Al analizar la clase funcional, el $72,7 \%$ de los pacientes co- 


\begin{tabular}{|c|c|}
\hline \multicolumn{2}{|c|}{ EDAD E ÍNDICE DE MASA CORPORAL } \\
\hline Variable & Resultado \\
\hline Edad (años) & $75,2+/-13$ \\
\hline Índice de masa corporal $\left(\mathrm{Kg} / \mathrm{m}^{2}\right)$ & $26,25+/-7,1$ \\
\hline \multicolumn{2}{|c|}{ SEXO } \\
\hline Variable & Resultado (\%) \\
\hline Masculino & $50,4(n=143)$ \\
\hline Femenino & $49,3(n=140)$ \\
\hline \multicolumn{2}{|c|}{ PROCEDENCIA } \\
\hline Variable & Resultado (\%) \\
\hline Imbabura & $70,4(n=200)$ \\
\hline Carchi & $18,4(n=52)$ \\
\hline Esmeraldas & $1,8(n=5)$ \\
\hline Pichincha & $2,5(n=7)$ \\
\hline Otros & $6,7(n=19)$ \\
\hline \multicolumn{2}{|c|}{ ETNIA } \\
\hline Variable & Resultado (\%) \\
\hline Blanca & $1,8(n=5)$ \\
\hline Mestiza & $73,9(n=209)$ \\
\hline Negra & $9,9(n=28)$ \\
\hline Indígena & $14,5(n=41)$ \\
\hline \multicolumn{2}{|c|}{ CLASE FUNCIONAL SEGÚN LA CLASIFICACIÓN NYHA } \\
\hline Variable & Resultado (\%) \\
\hline Clase 1 & $2,8(n=8)$ \\
\hline Clase 2 & $36(n=102)$ \\
\hline Clase 3 & $36,7(n=104)$ \\
\hline Clase 4 & $24,4(n=69)$ \\
\hline \multicolumn{2}{|c|}{ CONSUMO DE ALCOHOL } \\
\hline Variable & Resultado (\%) \\
\hline Sí & $14,8(n=42)$ \\
\hline No & $85,2(n=241)$ \\
\hline \multicolumn{2}{|c|}{ CONSUMO DE TABACO } \\
\hline Variable & Resultado (\%) \\
\hline Sí & $22,6(n=64)$ \\
\hline No & $77,4(n=219)$ \\
\hline \multicolumn{2}{|c|}{ ANTECEDENTE DE ENFERMEDAD PULMONAR OBSTRUCTIVA CRÓNICA } \\
\hline Variable & Resultado (\%) \\
\hline Sí & $22,3(n=63)$ \\
\hline No & $77,7(n=220)$ \\
\hline \multicolumn{2}{|c|}{ ANTECEDENTE DE ENFERMEDAD CEREBROVASCULAR ISQUÉMICA } \\
\hline Variable & Resultado (\%) \\
\hline Sí & $10,6(n=30)$ \\
\hline No & $89,4(n=253)$ \\
\hline \multicolumn{2}{|c|}{ ANTECEDENTE DE DIABETES } \\
\hline Variable & Resultado (\%) \\
\hline Sí & $16,3(n=46)$ \\
\hline No & $83,7(n=237)$ \\
\hline
\end{tabular}

\begin{tabular}{|c|c|}
\hline \multicolumn{2}{|c|}{ ETIOLOGÍA } \\
\hline Variable & Resultado (\%) \\
\hline Hipertensiva & $55,5(n=157)$ \\
\hline Isquémica & $20,8(n=59)$ \\
\hline Valvular & $3,5(n=10)$ \\
\hline Alcohólica & $5,7(n=16)$ \\
\hline Otras & $2,5(n=7)$ \\
\hline Desconocida & $12(n=34)$ \\
\hline \multicolumn{2}{|c|}{ TRATAMIENTO } \\
\hline Variable & Resultado (\%) \\
\hline IECA & $45,2(n=128)$ \\
\hline ARA2 & $23,2(n=65)$ \\
\hline Diurético de asa & $67,1(n=190)$ \\
\hline Betabloqueante & $51,2(n=145)$ \\
\hline Digoxina & $15,2(n=43)$ \\
\hline Antagonista de aldosterona & $39,2(n=111)$ \\
\hline \multicolumn{2}{|c|}{ VARIABLES HEMODINÁMICAS Y ECOCARDIOGRÁFICAS } \\
\hline Variable & Resultado \\
\hline Presión arterial sistólica $(\mathrm{mm}$ & $127,9+/-24$ \\
\hline Presión arterial diastólica (mr & $72,4+/-12$ \\
\hline Presión arterial media $(\mathrm{mm}$ & $90+/-15$ \\
\hline Presión de pulso $(\mathrm{mmHg}$ & $56+/-19$ \\
\hline Frecuencia cardiaca (latidos por & $81+/-15$ \\
\hline $\begin{array}{r}\text { Fracción de eyección reducid } \\
\text { ventrículo izquierdo (\%) }\end{array}$ & $49,5(95)$ \\
\hline $\begin{array}{l}\text { Fracción de eyección intermec } \\
\text { ventrículo izquierdo (\%) }\end{array}$ & $29,7(57)$ \\
\hline $\begin{array}{r}\text { Fracción de eyección conserva } \\
\text { ventrículo izquierdo (\%) }\end{array}$ & $20,8(40)$ \\
\hline TAPSE (mm) & $17,27+/-10,6$ \\
\hline \multicolumn{2}{|c|}{ PARÁMETROS LABORATORIALES } \\
\hline Variable & Resultado \\
\hline Hemoglobina (g/dL) & $13,5+/-3$ \\
\hline Hematocrito (\%) & $42,03+/-10$ \\
\hline Creatinina $(\mathrm{mg} / \mathrm{dL})$ & $1,51+/-1,4$ \\
\hline Filtrado glomerular (ml/min) & $53,2+/-27$ \\
\hline Sodio (mEq/L) & $132+/-31$ \\
\hline Potasio (mEq/L) & $4,1+/-1$ \\
\hline Pro-BNP (pg/ml) & $4983+/-2672$ \\
\hline Ácido úrico (mg/dL) & $4,41+/-3,8$ \\
\hline $\mathrm{TSH}(\mathrm{U} / \mathrm{L})$ & $4,01+/-10$ \\
\hline $\mathrm{T} 4$ (ng/dL) & $0,77+/-0,7$ \\
\hline Colesterol total (mg/dL) & $126+/-140$ \\
\hline Colesterol LDL (mg/dL) & $68+/-57$ \\
\hline Colesterol HDL (mg/dL) & $28+/-23$ \\
\hline Triglicéridos (mg/dL) & $94+/-85$ \\
\hline
\end{tabular}

Tabla 1. Características basales de los pacientes con insuficiencia cardíaca atendidos en el Hospital San Vicente de Paúl de la Ciudad de Ibarra entre los años 2015 y 2020.

rrespondieron a los grupos II y III. Este dato es diferente a los reportados previamente en los que la mayoría de los pacientes presentaron estadios más avanzados ${ }^{8,9}$ (Tabla 1). Esto podría explicarse por el hecho de que nuestro trabajo fue realizado con pacientes de un hospital general en el que se brinda atención a casos de leve y mediana complejidad, a diferencia de lo que sucede en hospitales de especialidad (Tabla 1).

Por otro lado, al analizar la fracción de eyección, el 49,5\% de los pacientes presentó valores de fracción de eyección redu-

cida (Tabla 1). Este hecho es semejante al encontrado en publicaciones anteriores ${ }^{10}$.

En lo referente al tratamiento, el 51,2\% de los pacientes fueron dosificados con beta bloqueadores (Tabla 1). En países desarrollados la prescripción de esta familia de medicamentos supera el 70\% lo que se asocia con la disminución de la mortalidad entre los pacientes con insuficiencia cardíaca ${ }^{6-9,11}$.

Al realizar el análisis de supervivencia en la muestra estudiada se evidenció que la sobrevida global fue del $17 \%$ a los 5 


\begin{tabular}{|c|c|c|c|c|c|c|c|}
\hline \multirow[b]{2}{*}{ Variable } & \multicolumn{6}{|c|}{ Etiología } & \multirow[b]{2}{*}{ Valor $\mathrm{p}$} \\
\hline & $\begin{array}{l}\text { Hipertensiva } \\
\qquad(\mathrm{n}=157)\end{array}$ & Isquémica (n=59) & $\begin{array}{c}\text { Valvular } \\
(\mathrm{n}=10)\end{array}$ & $\begin{array}{l}\text { Alcohólica } \\
(\mathrm{n}=16)\end{array}$ & $\begin{array}{c}\text { Desconocida } \\
(\mathrm{n}=\mathbf{3 4})\end{array}$ & $\begin{array}{l}\text { Otra } \\
(n=8)\end{array}$ & \\
\hline Edad (años) & $77,5+/-12$ & $74,2+/-10$ & $67,5+/-13$ & $67,9+/-14$ & $76,2+/-13$ & $55,7+/-23$ & 0 \\
\hline Índice de masa corporal $(\mathrm{Kg} / \mathrm{m} 2)$ & $26,4+/-7$ & $27,2+/-6$ & $25,5+/-6$ & $23,9+/-7$ & $24,7+/-6$ & $27,2+/-4$ & 0,447 \\
\hline Presión arterial sistólica (mmHg) & $133,4+/-23$ & $128,1+/-26$ & $112,9+/-25$ & $119,6+/-18$ & $111,8+/-13$ & $121,2+/-22$ & 0 \\
\hline Presión arterial diatólica (mmHg) & $74,3+/-13$ & $73,2+/-12$ & $59,5+/-12$ & $70+/-10$ & $67,5+/-10$ & $71,5+/-5$ & 0,002 \\
\hline Presión arterial media (mmHg) & $93,8+/-15$ & $90,1+/-18$ & $76,3+/-14$ & $86,1+/-10$ & $81,6+/-10$ & $88,2+/-9$ & 0 \\
\hline Presión de pulso (mmHg) & $59,6+/-19$ & $55,7+/-18$ & $55,1+/-22$ & $48,9+/-17$ & $45,9+/-13$ & $49,7+/-20$ & 0,002 \\
\hline Frecuencia cardiaca (latidos por minuto) & $83,6+/-15$ & $77,2+/-14$ & $82,1+-20$ & $79,7+/-13$ & $82,3+/-15$ & $83,4+/-11$ & 0,174 \\
\hline Hemoglobina $(\mathrm{g} / \mathrm{dl})$ & $13,6+/-3$ & $13,4+/-3$ & $12,2+/-4$ & $13,6+/-2$ & $13,7+/-3$ & $14,4+/-3$ & 0,784 \\
\hline Hematocrito (\%) & $42,4+/-10$ & $41,1+/-10$ & $37,4+/-14$ & $42+/-7$ & $42,5+/-10$ & $44,6+/-8$ & 0,664 \\
\hline Creatinina (mg/dl) & $1,60+/-1,4$ & $1,65+/-1,7$ & $1,08+/-0,6$ & $1,23+/-0,4$ & $1,2+/-1,1$ & $0,75+/-0,3$ & 0,289 \\
\hline Filtrado glomerular $(\mathrm{ml} / \mathrm{min})$ & $50,7+/-25$ & $51,9+/-29$ & $52+/-34$ & $66,4+/-27$ & $57,7+/-29$ & $69,8+/-42$ & 0,135 \\
\hline Sodio $(\mathrm{mEq} / \mathrm{l})$ & $134,1+/-29$ & $132,1+/-31$ & $124,9+/-44$ & $121,7+/-47$ & $131,7+/-33$ & $141,5+/-4$ & 0,639 \\
\hline Potasio $(\mathrm{mEq} / \mathrm{l})$ & $4,2+/-1,1$ & $4,2+/-1,1$ & $4,1+/-1,5$ & $3,7+/-1,2$ & $4+/-1,2$ & $4,3+/-0,3$ & 0,618 \\
\hline $\operatorname{ProBNP}^{*}(\mathrm{pg} / \mathrm{ml})$ & $6186,6+/-34118$ & $4688,9+/-17255$ & $3836,2+/-10969$ & $597,2+/-1222$ & $3105,5+/-6067$ & $1262,7+/-1992$ & 0,957 \\
\hline Ácido úrico (mg/dl) & $4,54+-3$ & $4,09+/-3$ & $4,60+/-4$ & $4,75+/-3$ & $3,89+/-4$ & $5,75+/-3$ & 0,82 \\
\hline $\mathrm{TSH}^{\ddagger}(\mathrm{UI} / 1)$ & $3,18+/-4$ & $5,9+/-19$ & $3,28+/-5$ & $0,9+/-1$ & $5,1+/-10$ & $8,8+/-12$ & 0,27 \\
\hline $\mathrm{fT} 4^{\uparrow}(\mathrm{ng} / \mathrm{dl})$ & $0,83+/-0,7$ & $0,77+/-0,7$ & $0,75+/-0,6$ & $0,39+/-0,6$ & $0,73+/-0,6$ & $0,62+/-0,5$ & 0,319 \\
\hline Colesterol total (mg/dl) & $130,9+/-173$ & $139,9+/-82$ & $98,5+/-91$ & $86,5+/-77$ & $96,3+/-80$ & $172,7+/-38$ & 0,456 \\
\hline Colesterol LDL (mg/dl) & $68,9+--59$ & $81,1+/-56$ & $65,9+/-60$ & $38,8+/-43$ & $52,7+/-54$ & $105,5+/-11$ & 0,026 \\
\hline Colesterol HDL (mg/dl) & $28,8+/-23$ & $31,1+/-22$ & $22,1+/-20$ & $16,8+/-16$ & $24,6+/-26$ & $52,2+/-24$ & 0,019 \\
\hline Trigliceridos (mg/dl) & $90,3+/-80$ & $123,3+/-100$ & $85,2+/-125$ & $76,4+/-74$ & $64,5+/-58$ & $154,5+/-77$ & 0,008 \\
\hline $\begin{array}{l}\text { Promedio de la fracción de eyección del } \\
\text { ventrículo izquierdo (\%) }\end{array}$ & $27+/-22$ & $32+/-14$ & $35+/-17$ & $27+/-25$ & $19+/-23$ & $33+/-20$ & 0,114 \\
\hline $\operatorname{TAPSE}^{\S}(\mathrm{mm})$ & $17,91+/-14$ & $17,72+/-3$ & $15,54+/-3$ & $13,8+/-4$ & $15,9+/-4$ & $14,6+/-2$ & 0,923 \\
\hline
\end{tabular}

Tabla 2. Diferencias entre las variables de los pacientes con insuficiencia cardíaca según la etiología de la enfermedad.

\begin{tabular}{|c|c|c|c|c|c|c|c|}
\hline \multirow{3}{*}{$\begin{array}{l}\text { Variable } \\
\text { Edad }\end{array}$} & \multirow{2}{*}{\multicolumn{2}{|c|}{$\begin{array}{l}\text { Comparación de subgrupos por } \\
\text { etiología }\end{array}$}} & \multirow{3}{*}{$\begin{array}{l}\begin{array}{l}\text { Diferencia } \\
\text { de medias }\end{array} \\
21,86533\end{array}$} & \multirow{3}{*}{$\begin{array}{l}\text { Error } \\
\text { estándar } \\
4,8711\end{array}$} & \multirow{3}{*}{$\begin{array}{l}\text { Valor } \mathrm{p} \\
0,00015\end{array}$} & \multicolumn{2}{|c|}{ Intervalo de confianza } \\
\hline & & & & & & \multirow{2}{*}{$\begin{array}{r}\begin{array}{c}\text { Límite } \\
\text { inferior }\end{array} \\
5,5396\end{array}$} & \multirow{2}{*}{$\begin{array}{c}\begin{array}{c}\text { Límite } \\
\text { superior }\end{array} \\
38,1911\end{array}$} \\
\hline & Hipertensiva & Otra & & & & & \\
\hline & Isquémica & Otra & 18,50605 & 5,0408 & 0,0213 & 1,6115 & 35,4006 \\
\hline & Desconocida & Otra & 20,55042 & 5,2337 & 0,01 & 3,0094 & 38,0914 \\
\hline Presión sistólica & Hipertensiva & Desconocida & 21,53803 & 4,3706 & 0,0003 & 6,8896 & 36,18964 \\
\hline $\begin{array}{l}\text { Presión } \\
\text { diastólica }\end{array}$ & Hipertensiva & Valvular & 14,81847 & 4,1212 & 0,0263 & 1,006 & 28,6309 \\
\hline $\begin{array}{c}\text { Presión arterial } \\
\text { media }\end{array}$ & Hipertensiva & Desconocida & 12,2414 & 2,8806 & 0,0035 & 2,587 & 21,8958 \\
\hline \multirow[t]{4}{*}{ Colesterol LDL } & Otra & Hipertensiva & 36,59691 & 6,4568 & 0,0001 & 16,7702 & 56,4236 \\
\hline & Isquémica & Alcohólica & 42,35699 & 13,1869 & 0,0336 & 2,2573 & 82,4566 \\
\hline & Otra & Alcohólica & 66,75893 & 11,7925 & 0,0002 & 29,5028 & 104,015 \\
\hline & Otra & Desconocida & 52,77731 & 10,3427 & 0,0001 & 21,7812 & 83,7734 \\
\hline Triglicéridos & Isquémica & Desconocida & 58,8516 & 16,4296 & 0,0071 & 11,0195 & 106,6837 \\
\hline Presión de pulso & Hipertensiva & Desconocida & 13,75703 & 3,523 & 0,0107 & 1,9496 & 25,5645 \\
\hline
\end{tabular}

Tabla S1. Análisis pos hoc de la diferencia entre variables de los pacientes con insuficiencia cardíaca según la etiología de la enfermedad.

años a partir del momento del diagnóstico (Figura 1). Este dato contrasta con estudios realizados en países desarrollados en los cuales este indicador oscila entre el $30 \%$ y el $60 \%$ durante el

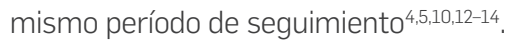

Al comparar nuestro resultado de sobrevida global con años pasados encontramos que este valor es incluso menor al reportado en la década de los años 50's donde la mortalidad a los 5 años a partir del momento del diagnóstico con insuficiencia cardíaca de manera independiente de la edad, del sexo y de la raza de los pacientes fue de aproximadamente igual al 70\%, para los años 90 se redujo al 59\% y a inicios de este siglo se ubicó en el 41\%9,10,13,15,16.

Varios estudios han descrito que diferentes factores están asociados con mayores tasas de mortalidad. Entre estos destacan: (i) la edad, (ii) el antecedente de cardiopatía isquémica, (iii) el padecer diabetes mellitus tipo 2, (iv) la presencia de proteinuria y/o síndrome cardiorrenal, (v) los niveles de nitrógeno ureico en sangre, (vi) la prescripción de antagonistas de aldosterona y (vii) la frecuencia cardíaca ${ }^{1,17,18}$. Frente a estos datos, el análisis de Cox en nuestra muestra demostró que la etiología hipertensi- 


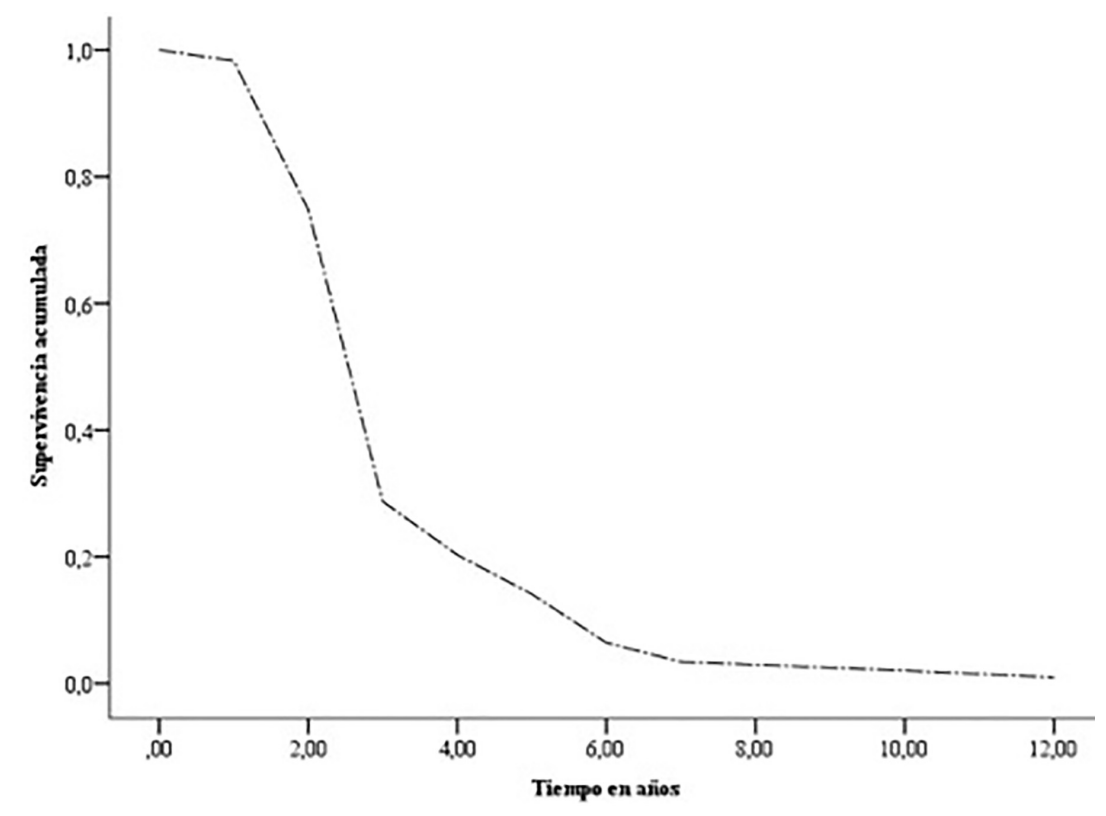

Figura 1. Curva de supervivencia acumulada de los pacientes diagnosticados con insuficiencia cardiaca entre los años 2015 y 2020.

Figura 2. Supervivencia ajustada de los pacientes con insuficiencia cardíaca en función de la etiología de la enfermedad.

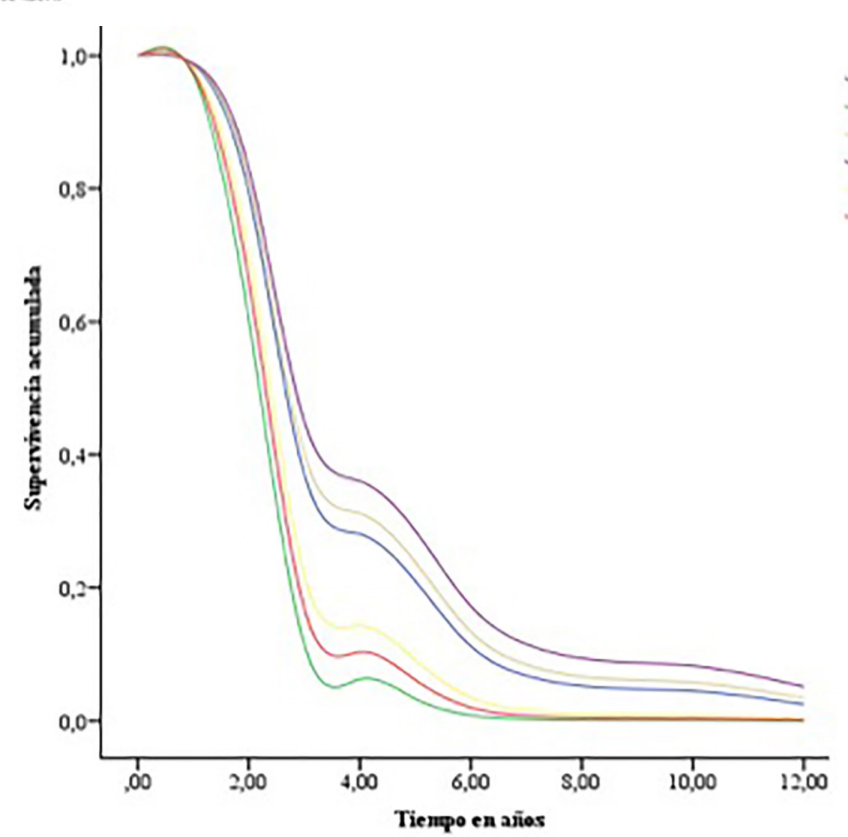

ETIOLOGLA HIPERTENSIVA
ISQUEMICA
VALVULAR VALVULAR ALCOHOLCA OTRA -DESCONOCIDA va y el menor número de hospitalizaciones son factores de protección lo que se traduce en menor mortalidad. Al contrario de lo que sucede con la disminución de valores del TAPSE.

Al realizar el análisis por subgrupos se encontró que el $20,8 \%$ de los casos de insuficiencia cardíaca fueron por enfermedad coronaria isquémica. Este hallazgo contrasta con estudios realizados en países del primer mundo donde la principal etiología fue la enfermedad coronaria isquémica, la cual es responsable de hasta el 30\% de los casos, sobre todo en hombres, con fracción de eyección preservada ${ }^{3.4}$.

En estudios semejantes, en países latinoamericanos, se han encontrado resultados heterogéneos por ejemplo, en trabajos publicados con muestras de Argentina y Brasil, entre el 30\% y el $40 \%$ de los diagnósticos de insuficiencia cardiaca fueron secundarios a enfermedad coronaria isquémica, mientras que en poblaciones de estrato económico más bajo como en el África Subsahariana la prevalencia de la enfermedad coronaria fue del $7,2 \%{ }^{17,19}$. Esta variación en los datos podría deberse a la diferencia de acceso a métodos diagnósticos más complejos como la coronariografía.

En relación a la causa hipertensiva, en nuestra muestra re- presentó la principal etiología de insuficiencia cardíaca con el $55,5 \%$ de los casos estudiados (Tabla 1 ). Lo que difiere de poblaciones de países desarrollados donde está causa oscila entre el $7 \%$ y el $13 \%$ de los casos de insuficiencia cardiaca ${ }^{3,19}$. Fisiopatológicamente, esto se explica porque la exposición prolongada de las células miocárdicas a niveles tensionales crónicamente elevados, resulta en la hipertrofia de ventrículo izquierdo y posterior disfunción, incluso en ausencia de enfermedad coronaria significativa o de infarto previo ${ }^{3}$.

El riesgo de presentar insuficiencia cardíaca por esta causa es el doble en pacientes masculinos y con valores tensionales promedio superiores a 160/90 mm Hg, lo que en términos de mortalidad se traduce en hasta el $76 \%$ de decesos a 5 años de seguimiento en ausencia de un tratamiento adecuado para la hipertensión arterial ${ }^{3,4}$. En cuanto a estudios realizados en poblaciones de países en vías de desarrollo, la proporción de casos de insuficiencia cardíaca asociados a enfermedad hipertensiva varió entre $20 \%$ y $84 \%{ }^{17,19}$, siendo similar a lo encontrado en este trabajo.

Por otro lado, es necesario considerar que la hipotensión persistente en pacientes con diagnóstico establecido de insufi- 
ciencia cardiaca es responsable del incremento de la letalidad en hasta el $18 \%$ por la reducción de $10 \mathrm{~mm} \mathrm{Hg}$ de la presión sistólica ${ }^{14}$.

La etiología valvular representa menos del 5\% de la totalidad de las causas de insuficiencia cardíaca. No obstante, en personas mayores de 75 años la enfermedad valvular degenerativa puede justificar hasta el $11,7 \%$ de insuficiencia cardíaca ${ }^{3}$. Además, la etiología valvular reumática en países desarrollados tiene una incidencia menor a 1 caso por 100000 habitantes. Sin embargo, en países del África Subsahariana la prevalencia de esta enfermedad puede ser de hasta el 13,8\% con una incidencia de hasta 100 casos por 100000 habitantes $^{3}$.

En este estudio la etiología valvular representó el 3,5\% de los casos de insuficiencia cardíaca, que es menor al reportado en países de renta media donde la prevalencia oscila entre el $6 \%$ y el $22 \%{ }^{19}$.

En cuanto a otras causas de la insuficiencia cardíaca se encuentran la: (i) miocardiopatía alcohólica, (ii) miocardiopatía periparto, y (iii) miocardiopatía dilatada idiopática que en conjunto representan entre el 3\% y el 30\% de las causas de esta enfermedad $^{19}$. En nuestro caso, estas etiologías constituyeron entre el 3\% y el 5\% de los casos estudiados. Sin embargo, no hay que descartar que estos valores podrían estar asociados a un alto porcentaje de subdiagnóstico.

Finalmente, y a diferencia de otros trabajos, nuestro estudio incluyó la categoría de insuficiencia cardiaca de causa desconocida, la cual representó el 12\% del total de diagnósticos y que se asoció a peor pronóstico vital.

\section{Conclusiones y recomendaciones}

La principal causa de insuficiencia cardiaca en la población estudiada fue la secundaria a la hipertensión arterial. El porcentaje de sobrevida a 5 años a partir del diagnóstico de insuficiencia cardíaca en los pacientes incluidos en este trabajo está muy por debajo de lo reportado en estudios realizados en países de renta media y alta.

La ausencia del diagnóstico nosológico influye directamente sobre el pronóstico vital de los pacientes. La carencia de una guía de práctica clínica de esta enfermedad influiría directamente sobre la alta mortalidad evidenciada en este estudio

\section{Limitaciones}

Al ser un estudio monocéntrico y realizado en un hospital general los resultados podrían no representar de manera adecuada los casos más complejos por lo que su diagnóstico puede estar infravalorado. Por otro lado, las carencias diagnósticas hacen que la definición etiológica de la insuficiencia cardiaca sea limitada, especialmente en lo concerniente a las causas isquémica e idiopática.

\section{Referencias bibliográficas}

1. Sarría-Santamera A, Prado-Galbarro FJ, Martín-Martínez MA, Carmona R, Gamiño Arroyo AE, Sánchez-Piedra C, et al. Supervivencia de pacientes con insuficiencia cardiaca en atención primaria. Aten Primaria. 2015;47(7):438-45. Available from: http://dx.doi. org/10.1016/j.aprim.2014.03.017

2. Laribi S, Aouba A, Nikolaou M, Lassus J, Cohen-Solal A, Plaisance $P$, et al. Trends in death attributed to heart failure over the past two decades in Europe. Eur J Heart Fail. 2012;14(3):234-9. Available from: http://dx.doi.org/10.1002/ejhf.1569

3. Ziaeian B, Fonarow GC. Epidemiology and aetiology of heart failure. Nat Rev Cardiol. 2016;13(6):368-78. Available from: http://dx.doi. org/10.1038/nrcardio.2016.25
4. Bui AL, Horwich TB, Fonarow GC. Epidemiology and risk profile of heart failure. Nat Rev Cardiol. 2011;8(1):30-41. Available from: http://dx.doi.org/10.1038/nrcardio.2010.165

5. Taylor CJ, Ordóñez-Mena JM, Roalfe AK, Lay-Flurrie S, Jones NR, Marshall T, et al. Trends in survival after a diagnosis of heart failure in the United Kingdom 2000-2017: population based cohort study. BMJ. 2019;364:1-10. Available from: http://dx.doi.org/10.1136/bmj.l223

6. Jiménez-Navarro MF, Ramirez-Marrero MA, Anguita-Sánchez M, Castillo JC. Influence of gender on long-term prognosis of patients with chronic heart failure seen in heart failure clinics. Clin Cardiol. 2010;33(3):13-8. Available from: http://dx.doi.org/10.1002/clc.20476

7. Franco J, Formiga F, Chivite D, Manzano L, Carrera M, Arévalo-Lorido JC, et al. New onset heart failure - Clinical characteristics and short-term mortality. A RICA (Spanish registry of acute heart failure) study. Eur J Intern Med [Internet]. 2015;26(5):357-62. Available from: http://dx.doi.org/10.1016/j.ejim.2015.04.008

8. Ocampo Chaparro JM, Badiel M, Casanova ME, Reyes-Ortiz CA, León Giraldo H, Castaño Cifuentes O. Características clínicas y supervivencia a 30 días de pacientes ancianos colombianos hospitalizados por insuficiencia cardiaca descompensada. Rev Esp Geriatr Gerontol. 2015;50(3):153-5. Available from: http://dx.doi. org/10.1016/j.regg.2014.11.013

9. Maggioni A Pietro. Epidemiology of Heart Failure in Europe. Heart Fail Clin [Internet]. 2015;11(4):625-35. Available from: http://dx. doi.org/10.1016/j.hfc.2015.07.015

10. Cho JH, Choe WS, Cho HJ, Lee HY, Jang J, Lee SE, et al. Comparison of characteristics and 3-year outcomes in patients with acute heart failure with preserved, mid-range, and reduced ejection fraction. Circ J. 2019;83(2):347-56. Available from: http://dx.doi. org/10.1253/circj.CJ-18-0543

11. Loh JC, Creaser J, Rourke DA, Livingston N, Harrison TK VE, Moriguchi J, Hamilton MA, Tseng C, Fonarow GC HT. Temporal Trends in Treatment and Outcomes for Advanced Heart Failure with Reduced Ejection Fraction from 1993-2010. Circ Hear Fail. 2013;6(3):411-9. Available from: http://dx.doi.org/10.1161/CIRCHEARTFAILURE.112.000178

12. Eriksson $B$, Wändell $P$, Dahlström $U$, Näsman $P$, Lund LH, Edner M. Comorbidities, risk factors and outcomes in patients with heart failure and an ejection fraction of more than or equal to $40 \%$ in primary care- and hospital care-based outpatient clinics. Scand J Prim Health Care [Internet]. 2018;36(2):207-15. Available from: https:// doi.org/10.1080/02813432.2018.1459654

13. Levy D, Kenchaiah S, Glarson M, Benjamin EJ, J.kupka M, Ho KKL, et al. Long-term trends in the incidence of and survival with heart failure. N Engl J Med. 2002;347(18):1397-402. Available from: https:// doi.org/10.1056/NEJMoa020265

14. Jumean MF, Kiernan MS. Determinants of survival following hospitalization for acute heart failure. Curr Heart Fail Rep. 2014;11(2):20111. Available from: https://doi.org/10.1007/s11897-014-0190-z.

15. Bytyçi I, Bajraktari G. Mortality in heart failure patients. Anadolu Kardiyol Derg. 2015;15(1):63-8. Available from: https://doi. org/10.5152/akd.2014.5731

16. Rodriguez F, Wang Y, Johnson CE, Foody JM. National patterns of heart failure hospitalizations and mortality by sex and age. J Card Fail. 2013;19(8):542-9. Available from: https://doi.org/ 10.1016/j. cardfail.2013.05.016

17. Agbor VN, Essouma M, Ntusi NAB, Nyaga UF, Bigna JJ, Noubiap JJ. Heart failure in sub-Saharan Africa: A contemporaneous systematic review and meta-analysis. Int J Cardiol [Internet]. 2018;257:207-15. Available from: https://doi.org/10.1016/j.ijcard.2017.12.048

18. Quiroz R, Doros G, Shaw P, Liang CS, Gauthier DF, Sam F. Comparison of characteristics and outcomes of patients with heart failure preserved ejection fraction versus reduced left ventricular ejection fraction in an urban cohort. Am J Cardiol [Internet]. 2014;113(4):691-6. Available from: http://dx.doi.org/10.1016/j.amjcard.2013.11.014

19. Bocchi EA. Heart Failure in South America. Curr Cardiol Rev. 2013:9(2):147-56. Available from: http://dx.doi. org/10.2174/1573403x11309020007

Received: 10 mayo 2020

Accepted: 1 julio 2020 